\title{
Exosomal miR-223 derived from natural killer cells inhibits hepatic stellate cell activation by suppressing autophagy
}

\author{
Ling Wang ${ }^{1}$, Yinghao Wang ${ }^{2}$ and Jun Quan ${ }^{1 *}$ D
}

\begin{abstract}
Background: Activation of hepatic stellate cells (HSCs) is a prominent driver of liver fibrosis. We previously demonstrated that exosomes derived from natural killer (NK) cells (NK-Exo) attenuated TGF- $\beta 1$-induced HSC activation. Herein, this study was designed to investigate the mechanism underlying the action of NK-Exo.

Methods: NK-Exo was isolated from NK-92MI cells and then administered into TGF- $\beta 1$-treated LX-2 (human HSC line) cells. MiR-223 expression in NK-Exo was downregulated by transfecting NK-92Ml cells with miR-223 inhibitor followed by exosome isolation. The HSC activation was evaluated by determining cell proliferation using CCK-8 assay and measuring the protein levels of a-SMA and CoL1A1 using western blot in LX-2 cells. The expression of miR-223 was detected by qRT-PCR. The interaction between miR-223 and ATG7 was analyzed by a dual-luciferase activity assay. The autophagy was evaluated by measuring the autophagy-related proteins using western blot.

Results: miR-223 was highly expressed in NK-Exo and inhibition of miR-223 expression in NK-Exo abrogated the inhibitory effect of NK-Exo on TGF- $\beta$-induced HSC activation. ATG7 was confirmed as a direct target of miR-223. Furthermore, treatment with the autophagy activator rapamycin and ATG7 overexpression in LX-2 cells abolished the HSC activation-suppressive effect of NK-Exo.
\end{abstract}

Conclusion: NK-Exo attenuated TGF- $\beta$-induced HSC activation by transferring miR-223 that inhibited autophagy via targeting ATG7.

Keywords: Hepatic stellate cell activation, Natural killer cell, Exosome, miR-223, Autophagy

\section{Background}

Activation of hepatic stellate cells (HSCs) is a prominent driver of liver fibrosis that can eventually lead to cirrhosis, liver failure, and even liver cancer (Higashi et al. 2017; Parola and Pinzani 2019). As such, effective therapeutic strategies for inhibiting HSC activation are urgently needed to reverse liver fibrosis. Exosomes are nano-sized membrane vesicles $(30-150 \mathrm{~nm}$ in diameter) that can be released from various cell types (Chen et al. 2020). Natural killer (NK) cells are important effector cells in many innate immune processes and play an

\footnotetext{
*Correspondence: qjun_af@163.com

'Department of Infectious Diseases, Xiangya Hospital of Central South

University, No. 87 Xiangya Road, Changsha 410008, Hunan, China

Full list of author information is available at the end of the article
}

important regulatory role in HSC activation (Fasbender et al. 2016; Foley et al. 2011). NK cells can influence the biological functions of recipient cells through secretion of exosomes (Shoae-Hassani et al. 2017; Neviani et al. 2019). Our group has previously demonstrated that exosomes derived from NK cells (NK-Exo) inhibited TGF$\beta 1$-induced HSC activation in HSC-LX-2 cells and carbon tetrachloride $\left(\mathrm{CCl}_{4}\right)$-induced liver fibrosis in BALB/ c mice (Wang et al. 2020a). However, the underlying mechanism of NK-Exo action remains unclear.

As a crucial means of intercellular communication, exosomes can transfer specific cargos including microRNAs (miRNAs) from the originating cells to the recipient cells (Zhang et al. 2019; Su et al. 2019). miRNAs are endogenous, small (19-22 nucleotides) RNA molecules that

(c) The Author(s). 2020 Open Access This article is licensed under a Creative Commons Attribution 4.0 International License, which permits use, sharing, adaptation, distribution and reproduction in any medium or format, as long as you give appropriate credit to the original author(s) and the source, provide a link to the Creative Commons licence, and indicate if changes were made. The images or other third party material in this article are included in the article's Creative Commons licence, unless indicated otherwise in a credit line to the material. If material is not included in the article's Creative Commons licence and your intended use is not permitted by statutory regulation or exceeds the permitted use, you will need to obtain permission directly from the copyright holder. To view a copy of this licence, visit http://creativecommons.org/licenses/by/4.0/. 
regulate gene expression through post-transcriptional pattern by directly binding to the 3 '-untranslated region ( 3 'UTR) of target mRNAs (Liu et al. 2018). Emerging evidence has indicated that miRNAs play a regulatory role in the occurrence and progression of liver fibrosis by influencing HSC activation (Zhao et al. 2019). miR-223 plays an essential role in the pathogenesis of various types of liver diseases, such as hepatitis virus infections, alcohol- or drug- induced liver injury, cirrhosis, and liver cancer (Ye et al. 2018). A recent study showed that treatment with miR-223-3p significantly mitigated fibrosis development and HSC activation in a murine model of fibrotic nonalcoholic steatohepatitis (NASH) (Jimenez Calvente et al. 2020).

Autophagy is a homeostatic, catabolic degradation process that degrades damaged cellular proteins and organelles to maintain cellular metabolism. Autophagy is induced during HSC activation and blockage of autophagy inhibits liver fibrosis by inhibiting HSC activation (Ye et al. 2020). Thus, autophagy may represent a potential target for developing anti-fibrotic strategies.

Autophagy-related 7 (ATG7), an autophagy marker, was identified as a putative target of miR-223 using Targetscan analysis (http://www.targetscan.org). Recently, Neviani et al. isolated NK cells from peripheral blood of healthy donors and profiled the top miRNAs represented in the exosomes. They found that miR-223 was highly expressed in NK cell-derived exosomes (Neviani et al. 2019). Thus, we hypothesized that NK cells might transfer miR-223 via exosomes to HSC-LX-2 cells where miR-223 suppressed autophagy via targeted inhibition of ATG7 expression, thereby attenuating TGF- $\beta 1$-induced HSC activation.

\section{Materials and methods}

\section{Cell culture}

The human NK cell line (NK92-MI; ATCC, Manassas, VA, USA) was cultured maintained in stem cell growth medium (Cellgro, Freiburg, Germany) containing 2\% exosome-depleted human serum and $1 \%$ penicillinstreptomycin. The human HSC line (LX-2; ATCC) was cultured in DMEM (Gibco, Grand Island, NY, USA) supplemented with 10\% FBS (Gibco), glutamine, sodium pyruvate, and $1 \%$ penicillin-streptomycin. Cells were maintained in a humidified air with $5 \% \mathrm{CO}_{2}$ at $37^{\circ} \mathrm{C}$.

\section{Cell transfection}

The miR-223 mimic, miR-223 inhibitor, mimic negative control (NC), inhibitor NC, ATG7 overexpression vector (pcDNA3.1-ATG7), and empty vector were purchased from GenePharma (Shanghai, China). NK-92MI cells at 70-80\% confluence were transfected with miR-223 inhibitor or inhibitor $\mathrm{NC}$ at a final concentration of 50 nM. LX-2 cells were transfected with these mimics, inhibitors, or vectors according to the experimental design. Cell transfection was performed using the Lipofectamine 3000 reagent (Invitrogen, Carlsbad, CA, USA). At $48 \mathrm{~h}$ post-transfection, cells were harvested for further assays.

\section{Isolation of exosomes from NK cells}

NK-92MI cells were used for exosome isolation after cultivation for 3 days according to a previous study (Zhu et al. 2017). When the cell confluence reached $70 \%$, the media were replaced with exosome-depleted FBS (Gibco). After incubation for $48 \mathrm{~h}$, cell supernatant was collected and centrifuged at $1500 \times \mathrm{g}$ for 3 $\mathrm{min}, 2000 \times \mathrm{g}$ for $15 \mathrm{~min}$, and then $3000 \times \mathrm{g}$ for $20 \mathrm{~min}$ at $4{ }^{\circ} \mathrm{C}$ to remove dead cells and cell debris. The supernatant was then centrifuged at $100,000 \times$ g for $1 \mathrm{~h}$ to obtain exosomes. The precipitated exosome pellets were resuspended in PBS and stored at $-80^{\circ} \mathrm{C}$ until further use. The exosomes derived from untreated NK-92MI cells, miR-223 inhibitor-transfected NK92MI cells, or inhibitor NC-transfected NK-92MI cells were referred to as NK-Exo, NK-Exo-miR-223I, or NK-Exo-NC, respectively.

\section{Characterization of exosomes}

The morphological characteristics of the exosomes were identified under transmission electron microscopy (TEM; Hitachi, Tokyo, Japan) as previously described (Yang et al. 2019). The nanoparticle tracking analysis was performed on a NanoSight NS500 (Malvern Instruments, Malvern, UK) to evaluate the size distribution of exosomes. Western blot was performed to detect the protein levels of exosomal surface markers (ALIX and CD63).

\section{Cell treatment}

LX-2 cells were treated with TGF- $\beta 1(5 \mathrm{ng} / \mathrm{mL})$ for $24 \mathrm{~h}$ to stimulate HSC activation. LX-2 cells in the NK-Exo treatment groups were pretreated with NK-Exo $(10 \mu \mathrm{g} /$ $\mathrm{mL}$ ) before TGF- $\beta 1$ treatment. LX-2 cells in the rapamycin treatment groups were pretreated with the autophagy activator rapamycin $(2 \mathrm{mM})$ in DMSO for $12 \mathrm{~h}$ before TGF- $\beta 1$ treatment.

\section{Luciferase reporter assay}

The interaction between miR-223 and 3'-UTR of ATG7 was verified by the luciferase reporter assay. Briefly, LX2 cells at $80 \%$ confluence were co-transfected with miR223 mimic/mimic NC, ATG7 wild-type (WT)/ATG7 mutated (Mut) luciferase constructs, and the internal control pRL-TK (Promega, Madison, WI, USA) using Lipofectamine 2000 (Invitrogen). At $24 \mathrm{~h}$ post-transfection, the luciferase activity was determined using a luciferase reporter assay system (Promega). 


\section{Cell proliferation assay}

LX-2 cells were seeded into 96-well plates at a density of $5 \times 10^{3}$ cells/well and were given different treatments. After $48 \mathrm{~h}$, the CCK- 8 reagent (Beyotime, Haimen, China) was added to each well and cells were incubated at $37^{\circ} \mathrm{C}$ for $4 \mathrm{~h}$. The $\mathrm{OD}_{450}$ values of different treatment groups were measured with a microplate detector (EnSpire 2300, PerkinElmer, Waltham, MA, USA).

\section{Quantitative real-time PCR (qRT-PCR)}

After different treatments, the cells or exosomes were lysed and total RNA was extracted using TRIzol reagent (Invitrogen). After reverse transcription, the expression of miR-223 was detected using the miRNA qRT-PCR kit (GeneCopoeia, Rockville, MD, USA) in Applied Biosystems 7500 PCR system (Applied Biosystems, Foster, CA, USA). The mRNA levels of $\alpha$-smooth muscle actin ( $\alpha$ SMA), collagen type I alpha 1 chain (CoL1A1) were detected using a LightCycler ${ }^{\circ} 480$ SYBR Green I Master qPCR mix (Roche, Mannheim, Germany). The specific primers were as follows: miR-223-Forward, 5' - CGTG TATTTGACAAGCTG - 3'; and miR-223-Reverse, 5'GAACATGTCTGCGTATCTC -3'; U6-Forward, 5' TGCGGGTGCTCGCTTCGCAGC- $-3^{\prime} ;$ and U6Reverse, 5' - CCAGTGCAGGGTCCGAGGT - 3'; $\alpha-$ SMA-Forward, 5' - GACAGCTACGTGGGTGACGAA $-3^{\prime}$; and $\alpha$-SMA-Reverse, 5' - TTTTCCATGTCGTC CCAGTTG - 3'; CoL1A1-Forward, 5' - GATTCC CTGGACCTAAAGGTGC - $3^{\prime}$; and CoL1A1-Reverse, 5' - AGCCTCTCCATCTTTGCCAGCA -3'; GAPDHForward, 5' - GTCTCCTCTGACTTCAACAGCG - 3'; and GAPDH-Reverse, 5' - ACCACCCTGTTGCTGTAG CCAA - 3'; Expression fold changes were calculated using the $2^{-\Delta \Delta \mathrm{Ct}}$ method. U6 was used as the reference gene for miR-223. GAPDH was used as the reference gene for $\alpha$-SMA and CoL1A1.

\section{Western blot}

Western blot analysis was performed as previously described (Wang et al. 2020b). After treatment, the cells or exosomes were lysed and total protein was extracted using the radioimmunoprecipitation assay lysis buffer (Beyotime). Proteins were electrophoresed by sodium dodecyl sulfate-polyacrylamide gel electrophoresis gels and transferred to polyvinylidene fluoride membranes (Millipore, Billerica, MA, USA). Then membranes were incubated overnight with primary antibodies against ALIX (1:1000, Abcam, Cambridge, MA, USA), CD63 (1: 1000; Abcam), $\alpha$-SMA (1:1000; Abcam), CoL1A1 (1: 1000; Abcam), p62 (1:1000; Santa Cruz Biotechnology, Dallas, TX, USA), Beclin-1 (1:1000, Santa Cruz Biotechnology), LC3 (1:1000; Santa Cruz Biotechnology), ATG7 (1:1000; Abcam), and GAPDH (1:3000; Abcam). The membranes were then incubated with the horseradish peroxidase-conjugated secondary antibodies (1:2000; Santa Cruz Biotechnology) for $1 \mathrm{~h}$ at room temperature.

\section{Statistical analysis}

SPSS 22.0 (IBM, Chicago, IL, USA) was used to perform statistical analyses. The student's $t$-test was used for comparison of two groups and ANOVA was used for multiple comparisons. $P<0.05$ was considered statistically significant.

\section{Results}

\section{Characterization of exosomes}

Exosomes were isolated from the conditioned medium derived from NK-92MI cells, namely NK-Exo. TEM micrographs showed that the isolated exosomes were spherical with a size distribution between $0 \sim 150 \mathrm{~nm}$ in diameter (Supplementary Fig. 1A and B). Furthermore, western blot analysis showed that the isolated exosomes positively expressed exosomal markers including ALIX and CD63 (Supplementary Fig. 1C).

\section{NK-Exo-mediated transfer of miR-223 attenuated TGF- $\beta 1$ - induced HSC activation}

Results of the qRT-PCR analysis showed that miR223 expression was markedly higher in NK-Exo than that in parental NK-92MI cells (Fig. 1a). To assess whether NK-Exo regulates HSC activation by transferring miR-223, we developed miR-223-deficient NKExo by transfecting NK-92MI cells with miR-223 inhibitor followed by exosomes isolation, namely, NKExo-miR-223I. miR-223 expression was confirmed to be sharply downregulated in NK-Exo-miR-223I when compared with the NK-Exo-NC group (Fig. 1b). TGF$\beta 1$ is important for HSC activation which is characterized by enhanced HSC cell proliferation and overproduction of CoL1A1 and $\alpha$-SMA (Zheng et al. 2016). NK-Exo treatment rescued the TGF- $\beta 1$ mediated downregulation of miR-223 expression in LX-2 cells (Fig. 1c). Of note, NK-Exo treatment significantly reduced the TGF- $\beta 1$-induced elevation of cell proliferation ratio (Fig. 1d) and upregulation of mRNA and protein levels of $\alpha$-SMA and CoL1A1 (Fig. 1e-f) in LX-2 cells. However, these effects of NK-Exo were counteracted when miR-223 expression in NK-Exo was inhibited (Fig. 1c-f). These results suggested that NK-Exo attenuated TGF- $\beta 1$-induced HSC activation by transferring miR-223.

NK-Exo-mediated transfer of miR-223 attenuated TGF- $\beta 1$ induced HSC activation by inhibiting autophagy

To determine whether autophagy is involved in exosomal miR-223-mediated attenuation of TGF- $\beta 1$-induced HSC activation, we examined several autophagy-related proteins using western blot in LX-2 cells treated as 


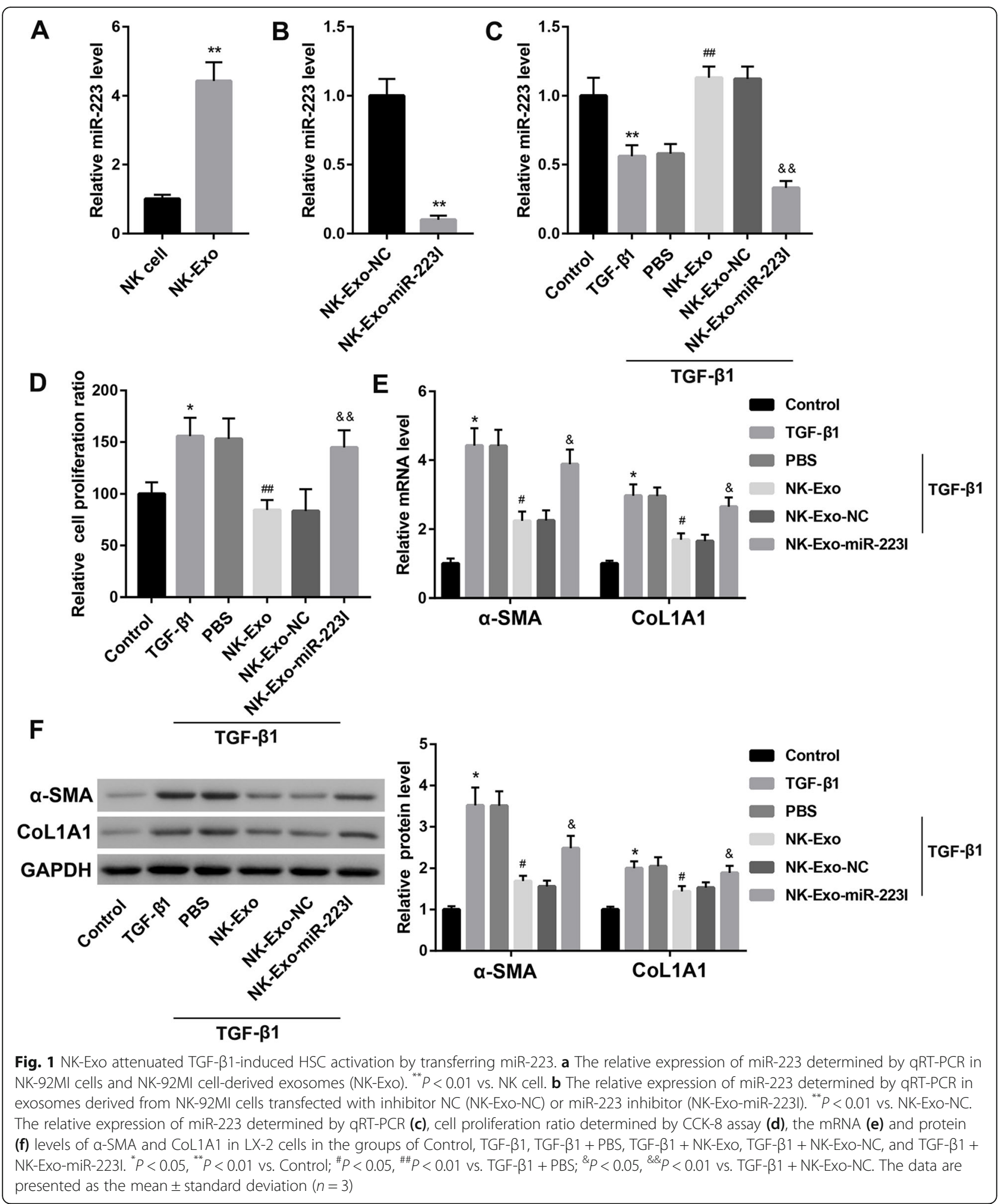

described above. NK-Exo treatment significantly increased p62 protein level, whereas decreased Beclin-1 protein level and LC3-II/LC3-I ratio in TGF- $\beta 1$ treated LX-2 cells. However, the autophagy-inhibitory effect of NK-Exo was compromised when miR-223 expression in NK-Exo was inhibited, suggesting that NK-Exo attenuated TGF- $\beta 1$-induced autophagy by transferring miR-223 (Fig. 2a). 


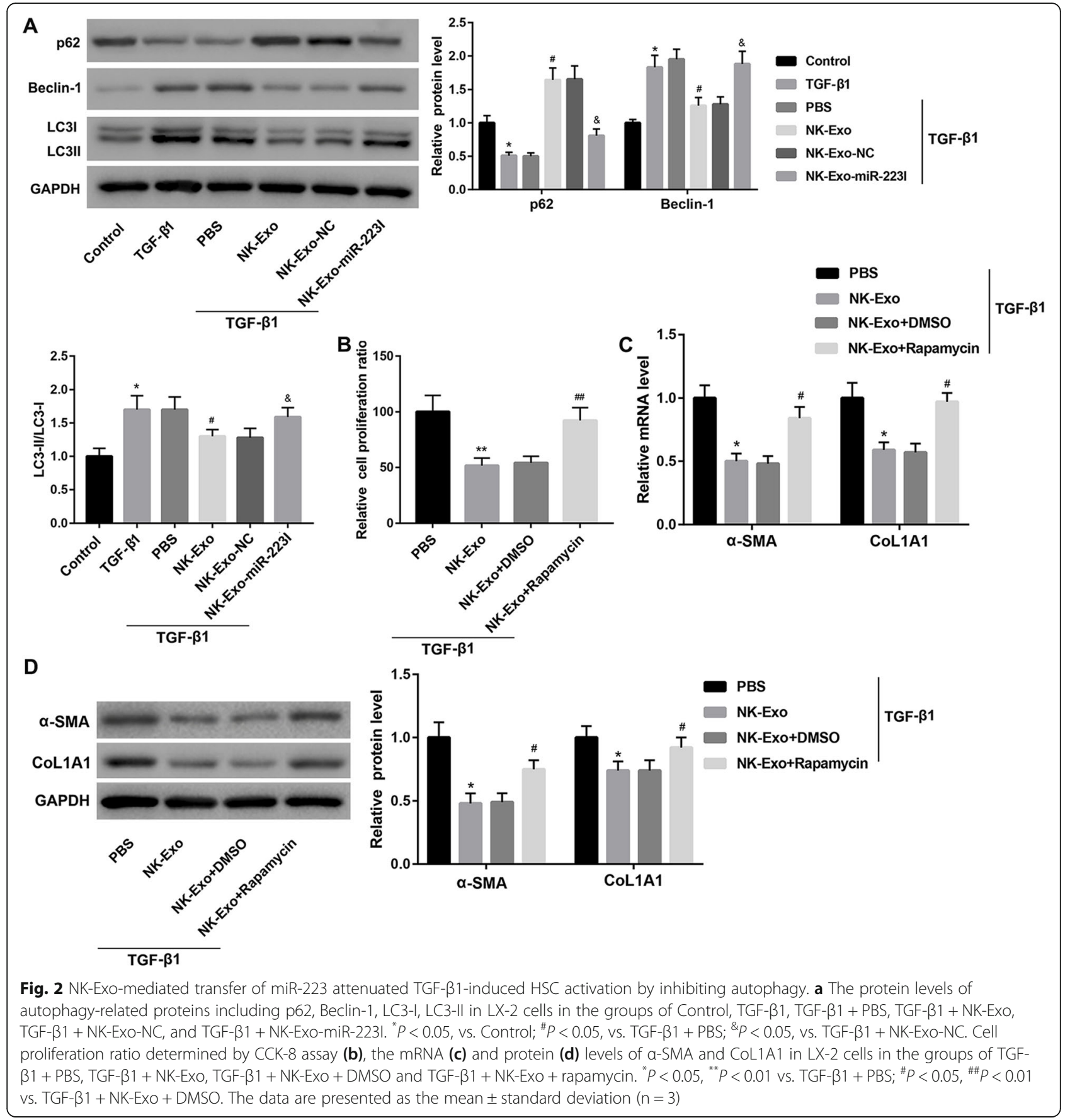

To further verify whether NK-Exo attenuates TGF$\beta 1$-induced HSC activation by inhibiting autophagy, we treated LX-2 cells with PBS or NK-Exo in the absence or presence of rapamycin (an autophagy activator) under TGF- $\beta 1$ stimulation. The cell proliferation ratio and the expression of $\alpha$-SMA and CoL1A1 were significantly upregulated in the TGF$\beta 1+$ NK-Exo + rapamycin group when compared with the TGF- $\beta 1+$ NK-Exo + DMSO group (Fig. 2b-d). Collectively, these data indicated that NK-Exo- mediated transfer of miR-223 attenuated TGF- $\beta 1$ induced HSC activation by inhibiting autophagy.

\section{NK-Exo-mediated transfer of miR-223 inhibited autophagy by targeting ATG7}

To explore the molecular mechanism by which exosomal miR-223 inhibits autophagy, we performed luciferase activity reporter assay to verify whether ATG7 is a downstream target of miR-223. Data revealed that miR223 mimic transfection significantly inhibited the 
luciferase activity of cells transfected with the ATG7 WT luciferase construct. Mutation of the binding sites could abolish the suppressing effect (Fig. 3a), verifying that miR-223 directly targeted ATG7.

NK-Exo treatment diminished the TGF- $\beta 1$-induced upregulation of ATG7 mRNA and protein levels in LX-2 cells. Inhibition of miR-223 expression in NK-Exo negated the suppressive effect of NK-Exo on ATG7 expression, indicating that NK-Exo inhibited ATG7 expression by transferring miR223 (Fig. 3b-c). To confirm the negative regulation of ATG7 expression in LX-2 cells by miR-223, we transfected LX-2 cells with miR-223 mimic and miR-223 inhibitor. The mRNA and protein levels of ATG7 were notably decreased following transfection with miR-223 mimic, whereas increased following transfection with miR-223 inhibitor (Fig. 3d-e).

\section{NK-Exo attenuated TGF- $\beta 1$-induced HSC activation by inhibiting ATG7 expression}

To examine the contribution of ATG7 to the suppressive effect of NK-Exo on HSC activation, we cultured

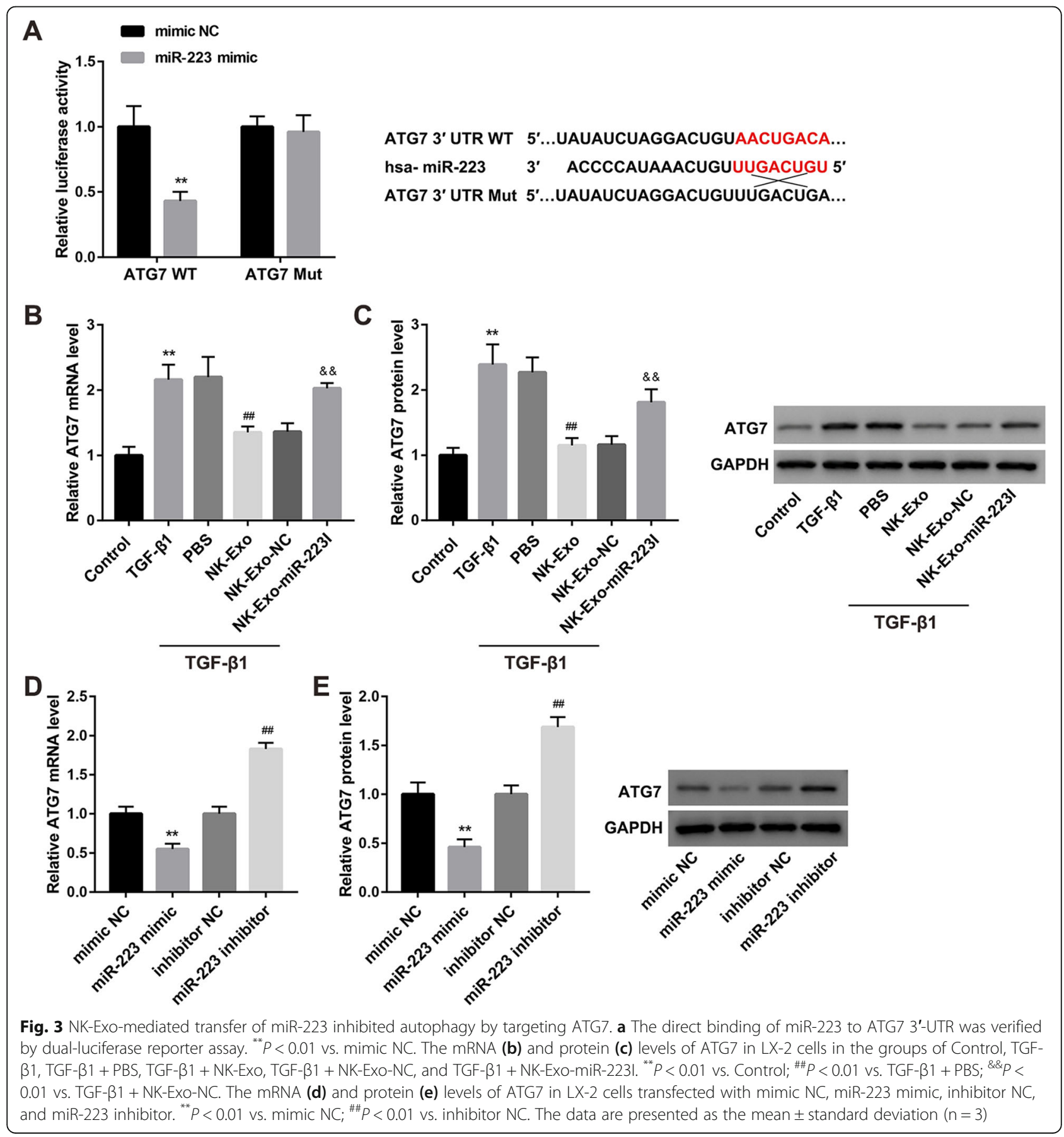


ATG7-overexpressing LX-2 cells with NK-Exo in the presence of TGF- $\beta 1$. Contrary to NK-Exo, ATG7 overexpression resulted in significant increases in cell proliferation ratio (Fig. 4a) and expression of $\alpha$-SMA and CoL1A1 (Fig. 4b-c) in LX-2 cells. Importantly, the inhibitory effects of NK-Exo on cell proliferation ratio (Fig. 4a) and expression of $\alpha$-SMA and CoL1A1 (Fig. 4bc) were abolished when ATG7 expression was overexpressed in LX-2 cells.

\section{Discussion}

HSCs are a prominent driver of liver fibrosis as the activated HSCs promote collagen deposition in the extracellular matrix by producing profibrogenic genes ( $\alpha$-SMA and CoL1A1), both of which are considered as markers of HSC activation (Yang et al. 2020). Thus it is essential to elucidate the mechanisms controlling HSC activation. TGF- $\beta 1$ is a canonical HSC activator after liver injury (Prestigiacomo et al. 2020). In the present study, we found that miR-223 was highly expressed in NK-Exo and inhibition of miR-223 expression in NK-Exo significantly abrogated the TGF- $\beta 1$-induced HSC activation, as demonstrated by suppressed proliferation and decreased expression of $\alpha$-SMA and CoL1A1 in human HSC-LX-2 cells. NK cell-derived exosomes can influence the biological functions of recipient cells by transferring miRNAs (Neviani et al. 2019). Our results showed that NKExo attenuated TGF- $\beta 1$-induced HSC activation by transferring miR-223.

Convincing evidence suggests that miRNAs are involved in regulating HSC activation and liver fibrosis. Zhou et al. demonstrated that miR-185 was lowly expressed in plasma of patients with hepatitis B virusrelated cirrhosis, livers of mice with $\mathrm{CCl}_{4}$-induced liver fibrosis, and TGF- $\beta 1$-activated HSC-LX- 2 cells. They also found that miR-185 overexpression inhibited HSC activation and hepatic fibrosis (Zhou et al. 2018). miR193a/b-3p, which was downregulated in liver tissues of

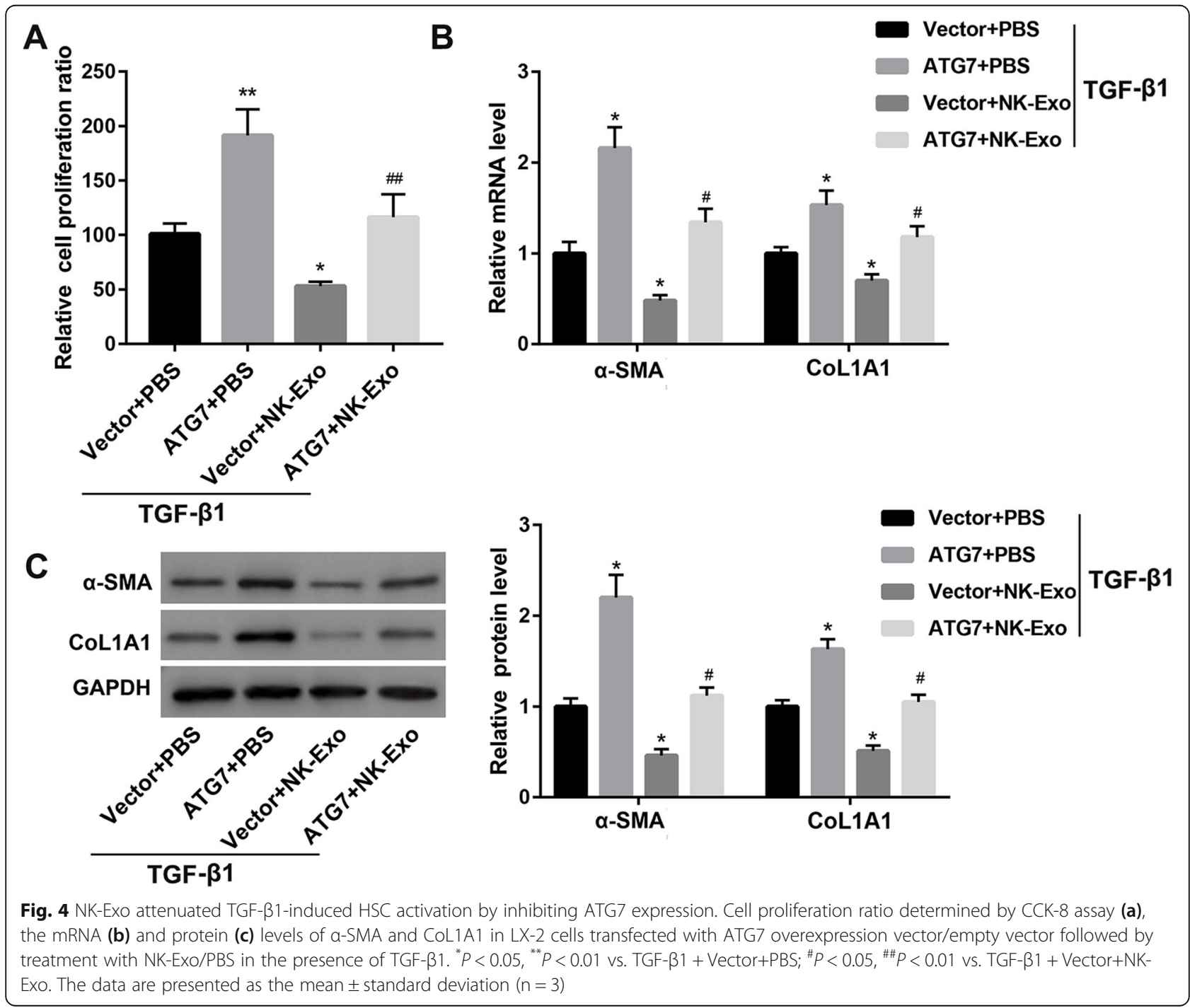


mice with concanavalin A-induced liver fibrosis, has been shown to relieve hepatic fibrosis through suppressing the proliferation and activation of HSCs (Ju et al. 2019). miR-223 is a miRNA involved in the pathogenesis of various liver diseases (Ye et al. 2018) and has been identified as a novel anti-inflammatory and anti-fibrotic therapeutic target (Calvente et al. 2019). Several studies have demonstrated that miR-223-3p alleviated hepatic fibrosis and suppressed HSC activation, confirming the hepatoprotective role of miR-223 (Jimenez Calvente et al. 2020; Liu et al. 2019a). Consistent with the antifibrotic effect of miR-223 described above, in this study, we experimentally confirmed that the inhibitory effect of NK-Exo treatment on TGF- $\beta$-induced HSC activation was mediated by miR-223 delivery.

A previous study showed that miR-223 inhibited hep3B (hepatocellular carcinoma cell line) cell proliferation and promoted apoptosis by directly targeting NLRP3 (NOD-like receptor family, pyrin domain containing 3) (Wan et al. 2018). Evidence indicates that activation of NLRP3 inflammasome can lead to HSC activation and liver fibrosis (Jiang et al. 2017; Inzaugarat et al. 2019). Therefore, we speculate that miR-223 may also attenuate HSC activation by inhibiting the NLRP3 inflammasome via targeting NLRP3. This speculation, however, awaits further validation.

The specific role of autophagy in HSC activation and liver fibrosis is sometimes contradictory. On one hand, autophagy induction has been shown to promote HSC activation and liver fibrosis. Ye et al. recently reported that ursodeoxycholic acid attenuated TGF- $\beta 1$-induced HSC activation and alleviated liver fibrosis in a rat model of $\mathrm{CCl}_{4}$-induced fibrosis by inhibition of autophagy (Ye et al. 2020). Also, Liu et al. proposed that isorhamnetin suppressed liver fibrosis and HSC activation in mouse models of liver fibrosis by reducing extracellular matrix formation and autophagy (Liu et al. 2019b). Consistently, the present study demonstrated that autophagy activation by rapamycin impaired the inhibitory effect of NK-Exo on TGF- $\beta$-induced HSC activation. Our findings manifested that autophagy is a profibrogenesis mechanism and autophagy inhibition may alleviate HSC activation and liver fibrosis. However, on the other hand, autophagy has also been suggested to protect against liver fibrosis. Insufficient autophagy has been implicated in liver fibrosis (Hazari et al. 2020). Ma et al. reported that ampelopsin, a natural flavonoid, attenuated $\mathrm{CCl}_{4}$-induced mouse liver fibrosis and HSC activation by inducing autophagy (Ma et al. 2019). Seo et al. found that Src inhibition increased autophagy flux and alleviated liver fibrosis and HSC activation (Seo et al. 2020). The protective effect of autophagy was contradictory to our results. Hence, the interactions between autophagy and HSC activation in the process of liver fibrosis are complicated due to diverse etiology and different types of cell settings, which require further investigation.

ATG7 is an autophagy marker. Yu et al. demonstrated that miR-96-5p prevented HSC activation by inhibiting autophagy via directly targeting ATG7 (Yu et al. 2018). In this study, ATG7 was confirmed as the direct target of miR-223 using the luciferase activity assay. Furthermore, ATG7 overexpression in HSC-LX2 cells abolished the inhibitory effect of NK-Exo on TGF- $\beta 1$-induced HSC activation. These results suggested that the mechanism by which NK-Exo attenuated TGF- $\beta$-induced HSC activation was related to exosomal miR-223 inhibition of autophagy via targeting ATG7.

In summary, the present study demonstrated for the first time that exosomal miR-223 derived from NK cells attenuated TGF- $\beta 1$-induced HSC activation by suppressing autophagy via targeted inhibition of ATG7. Regulation of miR-223 expression in exosomes released from NK cells might be a putative therapeutic strategy against liver fibrosis.

\section{Supplementary information}

Supplementary information accompanies this paper at https://doi.org/10. 1186/s10020-020-00207-w.

Additional file 1: Supplementary Figure 1. Characterization of NKExo. A. Morphological characterization of NK-Exo by TEM. B. The size distribution of NK-Exo was evaluated by the nanoparticle tracking analysis. C. The protein levels of the exosomal markers ALIX and CD63 were examined by western blot.

\section{Abbreviations}

3'-UTR: 3'-untranslated region; ATG7: Autophagy-related 7; $\mathrm{CCl}_{4}$ : Carbon tetrachloride; CoL1A1: Collagen type I alpha 1 chain; HSC: Hepatic stellate cell; miRNA: MicroRNA; Mut: Mutated; NASH: Nonalcoholic steatohepatitis; NC: Negative control; NK: Natural killer; NLRP3: NOD-like receptor family, pyrin domain containing 3; qRT-PCR: Quantitative real-time PCR;

TEM: Transmission electron microscopy; WT: Wild-type; a-SMA: a-smooth muscle actin

\section{Acknowledgements}

Not applicable.

\section{Authors' contributions}

Ling Wang performed the experiments and analyzed the data; Yinghao Wang interpreted the data and drafted the manuscript; Jun Quan designed the project and reviewed and edited the manuscript. The author(s) read and approved the final manuscript.

\section{Funding}

Not applicable.

\section{Availability of data and materials}

The datasets used and/or analysed during the current study are available from the corresponding author on reasonable request.

Ethics approval and consent to participate

All protocols were authorized by the Ethics Committee of Xiangya Hospital of Central South University.

Consent for publication

Not applicable. 


\section{Competing interests}

Not applicable.

\section{Author details}

'Department of Infectious Diseases, Xiangya Hospital of Central South University, No. 87 Xiangya Road, Changsha 410008, Hunan, China. 2Department of Ophthalmology, The Second Xiangya Hospital of Central South University, Changsha 410011, Hunan, China.

Received: 3 June 2020 Accepted: 28 July 2020

Published online: 01 September 2020

\section{References}

Calvente $\mathrm{CJ}$, et al. Neutrophils contribute to spontaneous resolution of liver inflammation and fibrosis via microRNA-223. J Clin Invest. 2019;130:4091-109.

Chen $L$, et al. Exosomal miR-103-3p from LPS-activated THP-1 macrophage contributes to the activation of hepatic stellate cells. FASEB J. 2020;34:517892.

Fasbender F, Widera A, Hengstler JG, Watzl C. Natural killer cells and liver fibrosis. Front Immunol. 2016;7:19.

Foley B, et al. NK cell education after allogeneic transplantation: dissociation between recovery of cytokine-producing and cytotoxic functions. Blood. 2011;118:2784-92.

Hazari Y, Bravo-San Pedro JM, Hetz C, Galluzzi L, Kroemer G. Autophagy in hepatic adaptation to stress. J Hepatol. 2020;72:183-96.

Higashi T, Friedman SL, Hoshida Y. Hepatic stellate cells as key target in liver fibrosis. Adv Drug Deliv Rev. 2017;121:27-42.

Inzaugarat $\mathrm{ME}$, et al. NLR family pyrin domain-containing 3 Inflammasome activation in hepatic stellate cells induces liver fibrosis in mice. Hepatology (Baltimore, Md). 2019;69:845-59.

Jiang $\mathrm{S}$, et al. Potentiation of hepatic stellate cell activation by extracellular ATP is dependent on P2X7R-mediated NLRP3 inflammasome activation. Pharmacol Res. 2017:117:82-93.

Jimenez Calvente C, Del Pilar H, Tameda M, Johnson CD, Feldstein AE. MicroRNA $2233 p$ negatively regulates the NLRP3 inflammasome in acute and chronic liver injury. Mol Therapy. 2020;28:653-63.

Ju B, et al. miR-193a/b-3p relieves hepatic fibrosis and restrains proliferation and activation of hepatic stellate cells. J Cell Mol Med. 2019;23:3824-32.

$\mathrm{Liu} \mathrm{H}$, et al. Nuclear functions of mammalian MicroRNAs in gene regulation, immunity and cancer. Mol Cancer. 2018;17:64

Liu N, et al. Isorhamnetin inhibits liver fibrosis by reducing autophagy and inhibiting extracellular matrix formation via the TGF-beta1/Smad3 and TGFbeta1/p38 MAPK pathways. Mediators Inflamm. 2019b;2019:6175091.

Liu W, Feng R, Li X, Li D, Zhai W. TGF-beta- and lipopolysaccharide-induced upregulation of circular RNA PWWP2A promotes hepatic fibrosis via sponging miR-203 and miR-223. Aging. 2019a;11:9569-80.

Ma JQ, et al. Ampelopsin attenuates carbon tetrachloride-induced mouse liver fibrosis and hepatic stellate cell activation associated with the SIRT1/TGFbeta1/Smad3 and autophagy pathway. Int Immunopharmacol. 2019;77: 105984.

Neviani P, et al. Natural killer-derived exosomal miR-186 inhibits neuroblastoma growth and immune escape mechanisms. Cancer Res. 2019;79:1151-64.

Parola M, Pinzani M. Liver fibrosis: pathophysiology, pathogenetic targets and clinical issues. Mol Aspects Med. 2019;65:37-55.

Prestigiacomo V, Weston A, Suter-Dick L. Rat multicellular 3D liver microtissues to explore TGF-beta1 induced effects. J Pharmacol Toxicol Methods. 2020;101: 106650.

Seo HY, et al. Src inhibition attenuates liver fibrosis by preventing hepatic stellate cell activation and decreasing connetive tissue growth factor. Cells. 2020;9: 558.

Shoae-Hassani A, et al. NK cell-derived exosomes from NK cells previously exposed to neuroblastoma cells augment the antitumor activity of cytokineactivated NK cells. J Immunotherapy (Hagerstown, Md : 1997). 2017;40:26576.

Su T, et al. Bone marrow mesenchymal stem cells-derived exosomal miR-29b-3p regulates aging-associated insulin resistance. ACS Nano. 2019;13:2450-62.

Wan L, Yuan X, Liu M, Xue B. miRNA-223-3p regulates NLRP3 to promote apoptosis and inhibit proliferation of hep3B cells. Exp Ther Med. 2018;15: 2429-35.

Wang L, Wang Y, Quan J. Exosomes derived from natural killer cells inhibit hepatic stellate cell activation and liver fibrosis. Hum Cell. 2020a;33:582-9.
Wang M, et al. Genipin inhibits the scleral expression of miR-29 and MMP2 and promotes COL1A1 expression in myopic eyes of Guinea pigs. Graefe's Arch Clin Exper Ophthalmol. 2020b.

Yang JJ, Yang Y, Zhang C, Li J, Yang Y. Epigenetic silencing of LncRNA ANRIL enhances liver fibrosis and HSC activation through activating AMPK pathway. J Cell Mol Med. 2020;24:2677-87.

Yang JX, Xie P, Li YS, Wen T, Yang XC. Osteoclast-derived miR-23a-5p-containing exosomes inhibit osteogenic differentiation by regulating Runx2. Cell Signal. 2019;70:109504.

Ye D, Zhang T, Lou G, Liu Y. Role of miR-223 in the pathophysiology of liver diseases. Exp Mol Med. 2018;50:128

Ye HL, et al. Ursodeoxycholic acid alleviates experimental liver fibrosis involving inhibition of autophagy. Life Sci. 2020;242:117175.

Yu K, et al. miR-96-5p prevents hepatic stellate cell activation by inhibiting autophagy via ATG7. J Mol Med (Berl). 2018;96:65-74.

Zhang X, et al. Hypoxic BMSC-derived exosomal miRNAs promote metastasis of lung cancer cells via STAT3-induced EMT. Mol Cancer. 2019;18:40.

Zhao Z, Lin CY, Cheng K. siRNA- and miRNA-based therapeutics for liver fibrosis. Transl Res. 2019;214:17-29.

Zheng J, et al. Long non-coding RNA PVT1 activates hepatic stellate cells through competitively binding microRNA-152. Oncotarget. 2016;7:62886-97.

Zhou L, et al. miR-185 inhibits fibrogenic activation of hepatic stellate cells and prevents liver fibrosis. Mol Ther Nucleic Acids. 2018;10:91-102.

Zhu $L$, et al. Exosomes derived from natural killer cells exert therapeutic effect in melanoma. Theranostics. 2017;7:2732-45.

\section{Publisher's Note}

Springer Nature remains neutral with regard to jurisdictional claims in published maps and institutional affiliations.

Ready to submit your research? Choose BMC and benefit from:

- fast, convenient online submission

- thorough peer review by experienced researchers in your field

- rapid publication on acceptance

- support for research data, including large and complex data types

- gold Open Access which fosters wider collaboration and increased citations

- maximum visibility for your research: over $100 \mathrm{M}$ website views per year

At BMC, research is always in progress.

Learn more biomedcentral.com/submissions 